\title{
A High Resolution Ultrawideband Wall Penetrating Radar
}

\author{
Erman Engin, Berkehan Çiftçioğlu, Meriç Özcan and İbrahim Tekin \\ Faculty of Engineering and Natural Sciences \\ Sabanci University, Tuzla, 34956 Istanbul, TURKEY
}

\begin{abstract}
A high resolution ultra wideband radar prototype is developed for through the wall imaging. The frequency range of operation of the radar is selected to be 1.85 to 6 $\mathrm{GHz}$ in order to have high spatial resolution. Besides the hardware, we have also developed a custom image processing software which attacks the problem of false target recognition and rejection. In this paper, we present our prototype along with various experimental results such as detecting stationary targets and detecting respiratory activity of a human behind a $23 \mathrm{~cm}$ thick brick wall.
\end{abstract}

Keywords: UWB Radar, Wall penetrating Radar, Time of arrival technique 


\section{INTRODUCTION}

In recent years, ultra wideband (UWB) radars have become very popular for military and medical applications. Several UWB radars have been developed for underground mine detection [1], [2], through the wall imaging [3], cancerous tissue detection applications [4], [5] with promising results. In addition to these imaging type applications, UWB signals are also used for material characterization [6].

UWB radar systems have significant advantages over traditional radar systems. The most important feature of an UWB radar system is the high spatial resolution as a result of employment of ultra narrow pulses [7]. Moreover, the power spectrum is spread over a wide frequency range, providing a low power density and reduced interference to other RF systems. Another advantage is that the UWB signals provide multipath immunity.

High resolution and high multipath immunity allows not only the detection of closely positioned targets, but also provides information about the shape and the material content of a target. As the employed bandwidth is increased, these advantages become more apparent. However, the trade-off is the difficulty of obtaining wideband components such as antennas, low noise amplifiers (LNA's), RF switches, UWB pulse generators and ultra fast signal digitizers. Wide bandwidth operation also causes additional challenges in signal processing. In general, one cannot stipulate the received signal shape and the traditional matched filter detection approach does not yield the optimum result for every target. The reason is that the reflected UWB pulse is distorted as the reflectivity of the target changes with the frequency.

In this paper, a high resolution UWB radar prototype for detecting targets behind the walls will be presented. There are reports of a few $\mathrm{GHz}$ bandwidth operating UWB systems, such as the ground penetrating radars (GPR) in [1], [8] (1 GHz bandwidth), the through wall radar presented in [3] (2 GHz bandwidth). The potential advantage of our UWB radar prototype is its wide operating bandwidth of $4 \mathrm{GHz}$ between 1.85 to $6 \mathrm{GHz}$.

The rest of the paper is organized as follows: In Section II, details of our prototype system including the individual RF components will be presented. In Section III, the signal processing algorithms to extract the useful information from the received signal, and the visualization algorithms for the display of the extracted data will be described. Later, the experiments performed for various types of stationary and moving targets will be presented in Section IV. Finally in Section V, the paper will be concluded with ideas for future work.

\section{UWB SYSTEM PROTOTYPE}

Our UWB system has two major building blocks, a transmitter section for generation and emission of the UWB pulses and a receiver section for capturing the 
reflected/scattered UWB signals from the target. A simplified block diagram of the system is shown in Figure 1. The prototype system currently has a single transmitter antenna, two receiving antennas placed on each side of the transmitting antenna in azimuthal plane, an UWB pulse generator, a low noise amplifier for each of the receiving antennas, a high speed sampling scope as part of the receiver, and a CPU for operation of the radar scenario and the signal processing.

A commercial pulse generator (Agilent 8133A) is used as the first component in the transmitter section for the generation of transmitted signals down to a pulse width of 100 picoseconds (psecs) with 3.3 Volts peak-to-peak amplitude. The generated pulse is then fed to the transmitter horn antenna, which has a gain of $9.2 \mathrm{dBi}$. The radiated signals from the horn antenna are reflected from the target and received by the two receiver antennas. The induced signals at each of the receiving antennas are then sent to LNA's which provide a gain of $26 \mathrm{~dB}$ between $100 \mathrm{MHz}$ and $6 \mathrm{GHz}$ with a very low noise figure of 1.3 $\mathrm{dB}$. The amplified signals are fed to the high speed sampling oscilloscope (Agilent $86100 \mathrm{C}$ ), where the samples are digitized. A personal computer (PC) controls the timing, the synchronization and the data collection from the high speed oscilloscope and performs the processing of the collected data to extract the location of the target. All of the algorithms for the data acquisition, the signal processing and the visualization are implemented in Matlab® software.

The receiving antennas employed in the UWB system prototype are custom designed microstrip-line-fed slot antennas with wideband operation. Both the antennas and the feeding structures are implemented on a $1.55 \mathrm{~mm}$ thick FR4 substrate with relative dielectric permittivity of 4.6. The measured input return losses $\left(\mathrm{S}_{11}\right)$ of the implemented receiving microstrip slot antennas are less than $-8 \mathrm{~dB}$ in the whole frequency range of 1.856.3 GHz as shown in Figure 2. The transmission response $\left(\mathrm{S}_{21}\right)$ of the microstrip slot antennas are measured by placing the two identical antennas facing each other in their far fields. The measured transmission response is plotted in Figure 3. Within the range of frequencies of interest, 1.85-6.3 GHz band, the slot antenna radiates reasonably well except with a null around $4.4 \mathrm{GHz}$, possibly caused by unwanted reflections from the measurement set-up. Besides the receiving antenna parameters, the other operating parameters of the radar are listed in Table I.

\section{SIGNAL PROCESSING ALGORITHMS}

The received signals after being sampled by the high speed scope have to be analyzed for target presence as well as the estimation of its location. Since the received signals are usually close to the noise level of the system, we have developed signal processing algorithms to detect the weak return signals. These algorithms maximize the signal to noise ratio (SNR) of the system, minimize the signal distortion and discard the undesired reflections. 
Our signal processing unit consists of three main modules: time of arrival (TOA) estimation module, calibration and background noise subtraction module, and the location calculation/visualisation module. In the TOA estimation module, the time of arrival from a possible target to one of the receiving antennas is estimated by applying envelope detection to the cross-correlation of the received signal with a locally generated UWB pulse synchronized to the transmitted pulse.

The purpose of the calibration and background noise subtraction module is to obtain a "cleaner" signal without any undesired echoes from the environment. Since the received signal levels are very low, in some scenarios when one has an access to the environment, it may be helpful to get a background scan to serve as a reference for the subsequent scans. This process not only helps to increase the SNR but also removes some of the deterministic undesired couplings between the transmitting and the receiving antennas. The location calculation/visualisation module includes the procedures of projecting the resultant signals after processing onto a two-dimensional x-y plane shown in Figure 4. In Figure 4, the transmitter (TX) antenna is located at $(0,0)$ and the two receiving antennas, $\mathrm{RX} 1$ and $\mathrm{RX} 2$, are located at $\left(-x_{r}, 0\right)$, and $\left(x_{r}, 0\right)$ respectively. Assume that there is a target located at $\left(x_{n}, y_{n}\right)$. When a signal is sent from the TX antenna, the receiver antennas pick up the signals which are reflected from the target as well as directly coming from the TX antenna. The signals picked up by the receiving antennas are first cross-correlated with the second derivative of Gaussian monopulse template signal. The reason for using the second derivative of the monopulse was that, in our case, it had the closest resemblance to the shape of the target echoes than any other derivative of the Gaussian monopulse. The cross-correlations of the received signals and the template signal are obtained as follows,

$$
\begin{aligned}
& R_{1}(\tau)=\int_{-\infty}^{\infty} x_{1}(t) \times z(t-\tau) d \tau \\
& R_{2}(\tau)=\int_{-\infty}^{\infty} x_{2}(t) \times z(t-\tau) d \tau
\end{aligned}
$$

where $z(t)$ is the second derivative of Gaussian monopulse, and $x_{1}(t)$ and $x_{2}(t)$ are the two received signals. The next step for enhancing the visibility of the cross-correlated signals is the envelope detection which can be expressed as,

$$
s_{i}(t)=\left(R_{i}(t)\right)^{2} \otimes h_{f}(t)
$$

where $\otimes$ is the convolution operator, $h_{f}(t)$ is the impulse response of the low pass filter and $i=1,2$ for the two cross-correlated signals. After the envelope detection of the cross correlated signals, the resultant samples can be manipulated to find the position of the targets. For this purpose, an x-y plane is meshed into square cells as shown in Figure 4, and the location mapping algorithm is applied as follows: given any cell on the $\mathrm{x}-\mathrm{y}$ plane, the total path length from the TX antenna to the cell and from that cell back to one of the 
RX antennas is calculated. For example, the total path length for a cell with the center coordinates $\left(x_{n}, y_{n}\right)$ for the two receiver antennas are given by,

$$
\begin{aligned}
& D_{n, 1}=L_{n, 1}+L_{n, 2}=\sqrt{x_{n}^{2}+y_{n}^{2}}+\sqrt{\left(x_{n}+x_{r}\right)^{2}+y_{n}{ }^{2}} \\
& D_{n, 2}=L_{n, 1}+L_{n, 3}=\sqrt{x_{n}^{2}+y_{n}^{2}}+\sqrt{\left(x_{n}-x_{r}\right)^{2}+y_{n}^{2}}
\end{aligned}
$$

This procedure of calculating path length is repeated for each cell in the x-y plane and for each receiving antenna and the calculated values are stored in a position matrix. Hence, each cell is identified with its two total path lengths to the receiving antennas. The total path lengths can be transformed into TOA values which are given by,

$$
t_{n, i}=\frac{D_{n, i}}{c}
$$

where $\mathrm{c}$ is the speed of light in the propagation medium and $i=1,2$ for the two crosscorrelated signals. For each cell in Figure 4, we use a weight factor for each receiving antenna. This weight factor is determined from the cross-correlated and envelope detected samples by substituting Eqn. (6) into Eqn. (3). More specifically, for a cell located at $\left(x_{n}, y_{n}\right)$, the weight factor for the first receiving antenna is given by $s_{1}\left(t_{n, 1}\right)$ and for the second receiving antenna, the weight is $s_{2}\left(t_{n, 2}\right)$. We define the likelihood of a target being in the cell $\left(x_{n}, y_{n}\right)$ as the total weight factor which is,

$$
w_{n}=s_{1}\left(t_{n, 1}\right) \times s_{2}\left(t_{n, 2}\right)
$$

As an estimation of the location of the target, the cells with the highest weights are chosen as the solutions. As an example, two sample received signals from both of the antennas are shown in Figure 5a and 5b where there is a single target present. In these figures, it is not possible to locate the echoes coming from the target. Especially, in an environment where there are number of objects, the received signals may include many reflections, which makes it challenging to resolve the real target echoes. However, as the calibration is performed, the target reflections can be clearly observed in Figures $5 \mathrm{c}$ and $5 \mathrm{~d}$ for the two receiving antennas. The calibration signals are obtained by storing the two received signals when there is no target in the environment. If a target is present and if the received signal is calibrated with the previously stored signals, the reflections from the unwanted objects are eliminated, leaving only the reflection from the target. Also note that for moving targets, detection of the target reflections are easier since we can look at the difference of two consecutive snapshots of the received signals, and hence remove the undesired stationary reflections. One can see the final waveforms after the process of correlation, calibration, and envelope detection in Figure 6. The peaks which correspond to TOAs of the target is obviously around $8 \mathrm{nsec}$ for the receiving antenna 1 , and around $8.5 \mathrm{nsec}$ for the receiving antenna 2. 
As a last step in the algorithms module, the TOAs should be converted into weights using Equation (7), and then mapped on to the $\mathrm{x}-\mathrm{y}$ plane with the intensity proportional to the weights. For the above example, when this procedure is performed for a $300 \mathrm{~cm} \mathrm{x}$ $300 \mathrm{~cm} x-y$ plane with 90,000 cells, processed waveforms yield the weights as plotted in Figure 7 . The target return is clearly seen as the bright circled region. Also note that, ellipses in the display are the loci of constant TOAs.

In the case of multiple targets and the multiple receiving antennas, it is possible to obtain false targets due to the intersection of constant TOA ellipses. As it can be seen in Figure 8, there are two false targets in addition to the two real targets. In some scenarios, we can get rid of the false target images by taking two snapshots of the environment at two different orientations of the antennas, and by properly combining these snapshots. We present such an example in the next section.

\section{EXPERIMENTAL RESULTS}

In this section, we present three main experiments which show the performance of our UWB radar. In the first experiment, tracking capability of the UWB radar is demonstrated qualitatively. In the second experiment, respiratory activity of a human behind a wall is observed. In the last experiment, elimination of the false target problem is investigated.

\section{Experiment 1:}

In our first experiment, a plastic ball with $12 \mathrm{~cm}$ radius covered with aluminium foil was located at a distance of 1.45 meters from the TX antenna and it was tied to the ceiling via a thin rope. As the covered ball swung, an oscillatory movement with a period of $3 \mathrm{sec}$ was observed experimentally. The waveforms received by a single receiver antenna were acquired with a sampling period of $380 \mathrm{msec}$. The acquired data vectors were concatenated to form a matrix, which is plotted in Figure 9. As it can be seen from the bright region in the figure, motion of the target can be tracked with a reasonable resolution.

\section{Experiment 2:}

As the next experiment, our aim was to test our system for detection of a live person behind a wall, which might be the case for a survivor in earthquake rubble or for surveillance by peace keeping forces. We placed our radar in front of a $23 \mathrm{~cm}$ thick brick wall behind which there was a stationary human target. We aimed to detect the breathing motion of the human target. The UWB radar is operated at a sampling period of 380 msec. The resultant waveforms are concatenated and plotted to form a 3D surface, as shown in Figure 10. The $\mathrm{x}, \mathrm{y}, \mathrm{z}$ axes in the figure correspond to snapshot number (time), TOA, and the amplitude of the calculated weights, respectively. The ripples in the amplitude with respect to time occur due to the respiratory activity of the human target. 
As the chest expands and collapses, the position of the related echoes shift in time. If an $\mathrm{X}-\mathrm{Z}$ cut is taken through the amplitude peaks of Figure 10, the periodic breathing motion is clearly seen as shown in Figure 11.

\section{Experiment 3:}

In this experiment, two targets were placed behind a $2.5 \mathrm{~cm}$ thick wooden panel to investigate the false target problem and to check whether our algorithms can differentiate between the real target and the false target. One of the targets was the plastic ball with $12 \mathrm{~cm}$ radius and the other was a cylindrical bottle each covered with aluminium foil. In Figure 12, view of the UWB radar display without our false target removal algorithm applied is shown. It is seen in the figure that there are real targets detected as well as false targets. However, when we apply our false target removal algorithm, which requires the view of the scene from an additional antenna orientation and proper combination of these two views, we make these false targets disappear as shown in Figure 13. In this experiment, we have obtained the second view by mechanically rotating the antenna assembly by an angle of 15 degrees in azimuthal plane.

\section{CONCLUSION}

In this paper, we have presented an UWB radar prototype for through-the wall imaging operating between $1.85 \mathrm{GHz}$ and $6 \mathrm{GHz}$ with two custom built receiving antennas and one transmitting horn antenna. The system has a peak output power of $110 \mathrm{~mW}$ and the maximum unambiguous range of the radar is $4.5 \mathrm{~m}$. Several experiments were conducted to analyze the performance of the radar. In one of the experiments, the respiratory activity of a human behind a $23 \mathrm{~cm}$ thick brick wall has been detected successfully. Also, a solution for false target rejection is proposed and it was shown to work experimentally when multiple targets were involved. As future work, two additional antennas will be placed vertically in order to map the targets in three dimensions.

\section{REFERENCES}

1. J. Homer, H. T. Tang, and I. D. Longstaff, Radar imaging of shallow buried objects, In: Proceedings of IEEE International Geoscience and Remote Sensing Symposium, IEEE, 1999, 2477-2479.

2. G. C. Gaunaurd and L. H. Nguyen, Detection of land-mines using ultra-wideband radar data and time-frequency signal analysis, In: Proceedings of IEE Radar Sonar Navig, IEE, 2004, 307-316.

3. S. Nag, H. Fluhler, and M. Barnes, Preliminary interferometric images of moving targets obtained using a time-modulated ultra-wide band through-wall penetration 
radar, In: Proceedings of IEEE Radar Conference, Atlanta, GA, IEEE, 2001, 6469.

4. S. K. Davis, H. Tandradinata, S. C. Hagness, and B. D. Van Veen, Ultrawideband Microwave Breast Cancer Detection: A Detection-Theoretic Approach Using the Generalized Likelihood Ratio Test, IEEE Trans Biomed Eng 52 (2005), 12371250 .

5. X. Li, S. K. Davis, S. C. Hagness, D. W. van der Weide, and B. D. Van Veen, Microwave imaging via space-time beamforming: experimental investigation of tumor detection in multilayer breast phantoms, IEEE Trans Micro Theory Tech MTT-52 (2004), 1856-1865.

6. V. Z. Marmarelis, D. Sheby, E. C. Kisenwether, T. A. Erdley, Target material characterization using high-order signal processing of ultra-wideband radar data, In: Proceedings of IEEE National Telesystems Conference, Washington, D.C., IEEE, 1992, 1/17 - 1/24.

7. I. Immoreev, Ten Questions about UWB, IEEE Aerospace and Electronic Systems Magazine 18-11 (2003), 8-10.

8. G. F. Stickley, I. D. Longstaff, and M. J. Radcliffe, Synthetic aperture radar for the detection of shallow buried objects, In: Proceedings of EUREL International Conference on The Detection of Abandoned Land Mines: A Humanitarian Imperative Seeking a Technical Solution, London, UK, IEE, 1996, 160-163. 


\section{Figures}

Figure 1. The block diagram of the system: pulse generator, TX/RX antennas, the scope, and LNAs.

Figure 2. The return loss (S11) of one of the receiving antennas.

Figure 3. The transmission parameter (S21) of the two identical microstrip slot antennas.

Figure 4. The position of the TX/RX antennas and the target in the $\mathrm{x}-\mathrm{y}$ planes.

Figure 5. The received and calibrated waveforms. a) by receiver-1 b) by receiver- 2 c) by receiver-1 after calibration d) by receiver-2 after calibration.

Figure 6. Waveforms after signal processing with the peaks corresponding to TOAs of the target.

Figure 7. Weights are mapped to an intensity plot on an $x-y$ plane $(300 \mathrm{~cm} \times 300 \mathrm{~cm}$ with $1 \mathrm{~cm}$ grid). The bright region shows the position of the target.

Figure 8. The real and false targets obtained by the intersection of four ellipses.

Figure 9. Intensity plot of the swinging ball with the period of the oscillatory motion as $3.04 \mathrm{sec}$.

Figure 10. A 3-D intensity plot when a human is breathing behind a $23 \mathrm{~cm}$ thick brick wall with the ripples representing the respiratory activity.

Figure 11. The respiration profile with the estimated $3.5 \mathrm{sec}$ period.

Figure 12. The intensity plot under multiple targets where a bottle and a metallic ball are placed in front of the radar.

Figure 13. Elimination of the false targets by combining the results of two different positions of the antennas.

\section{Tables}

Table 1: Radar operating parameters. 


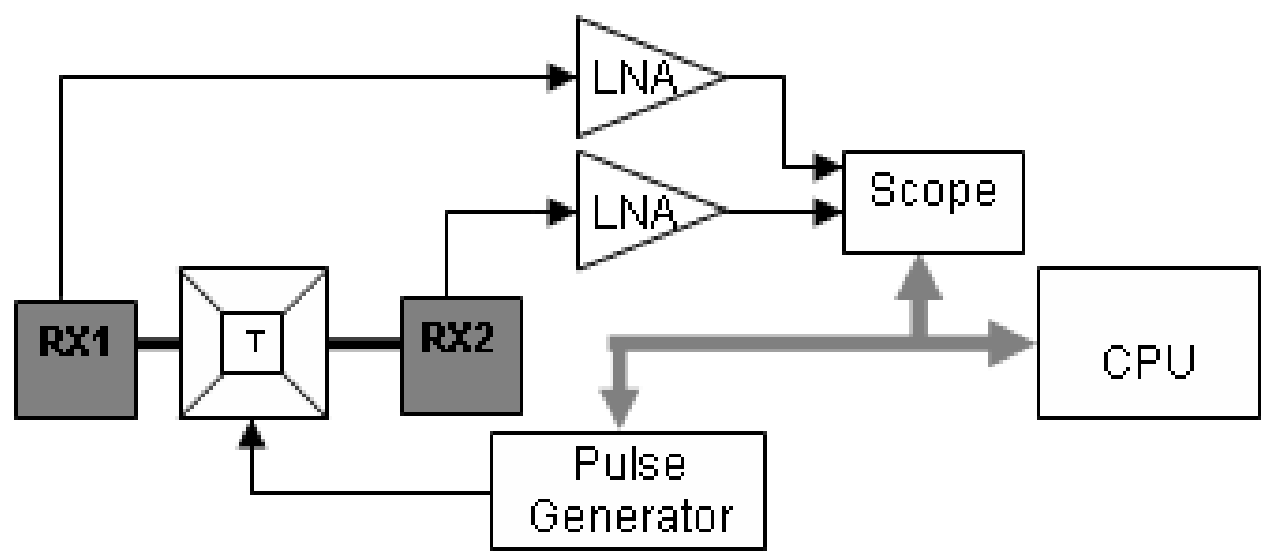

Figure 1: The block diagram of the system: pulse generator, TX/RX antennas, the scope, and LNAs. 


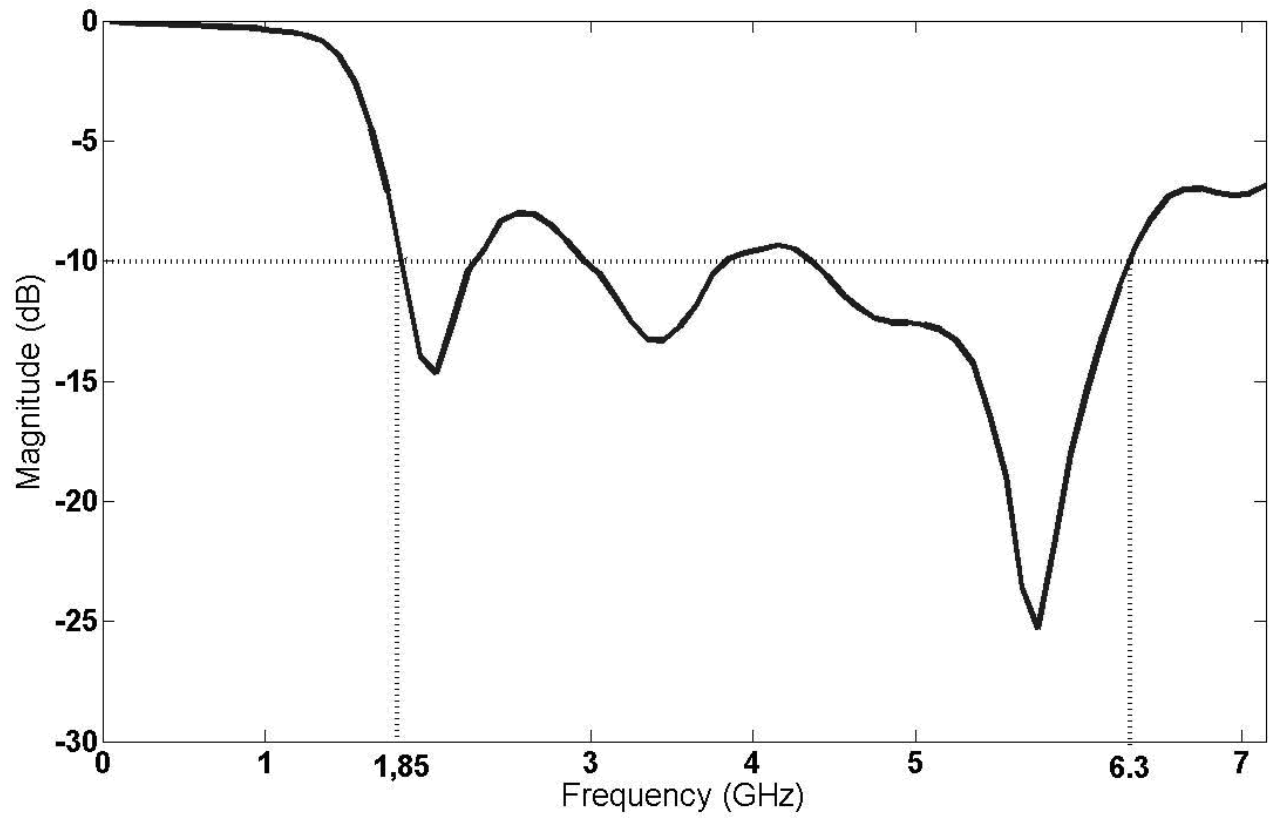

Figure 2: The return loss (S11) of one of the receiving antennas. 


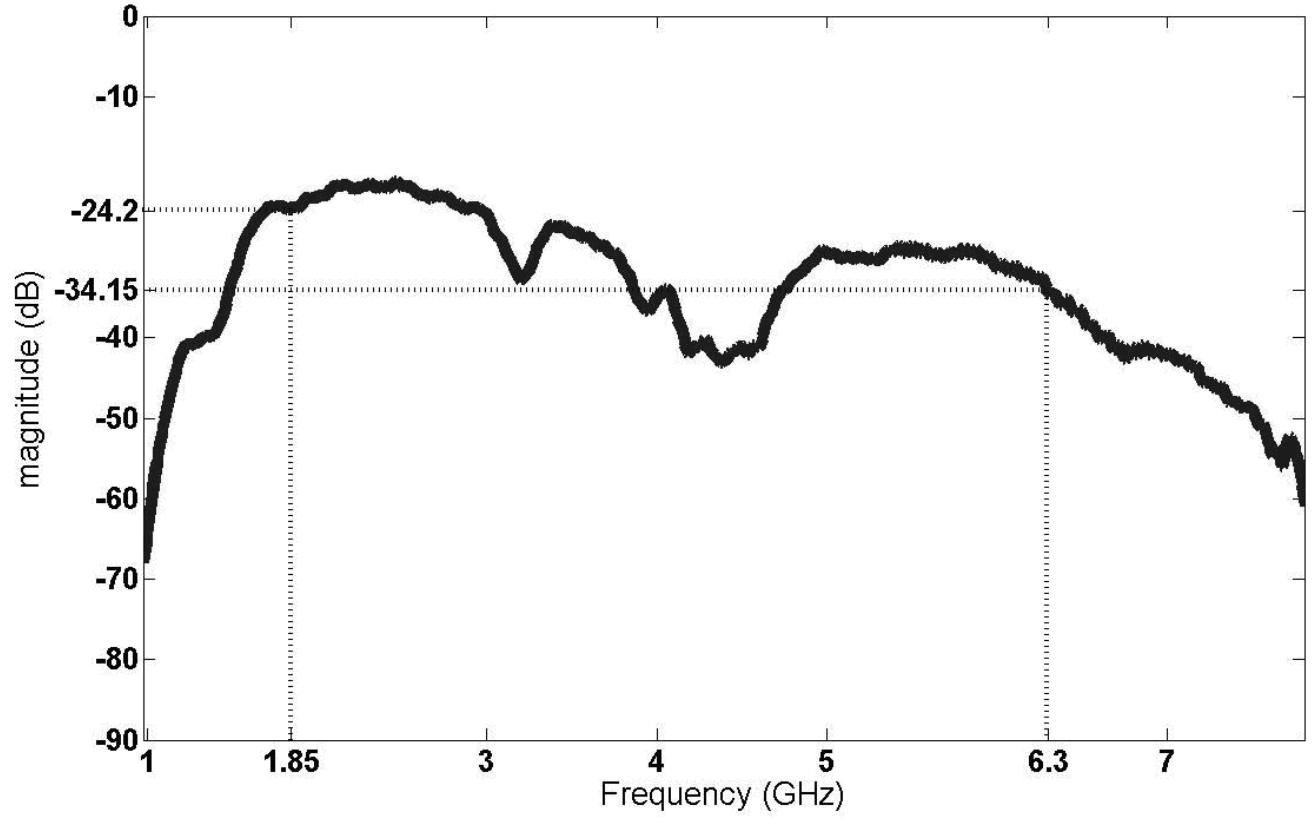

Figure 3: The transmission parameter (S21) of the two identical microstrip slot antennas. 


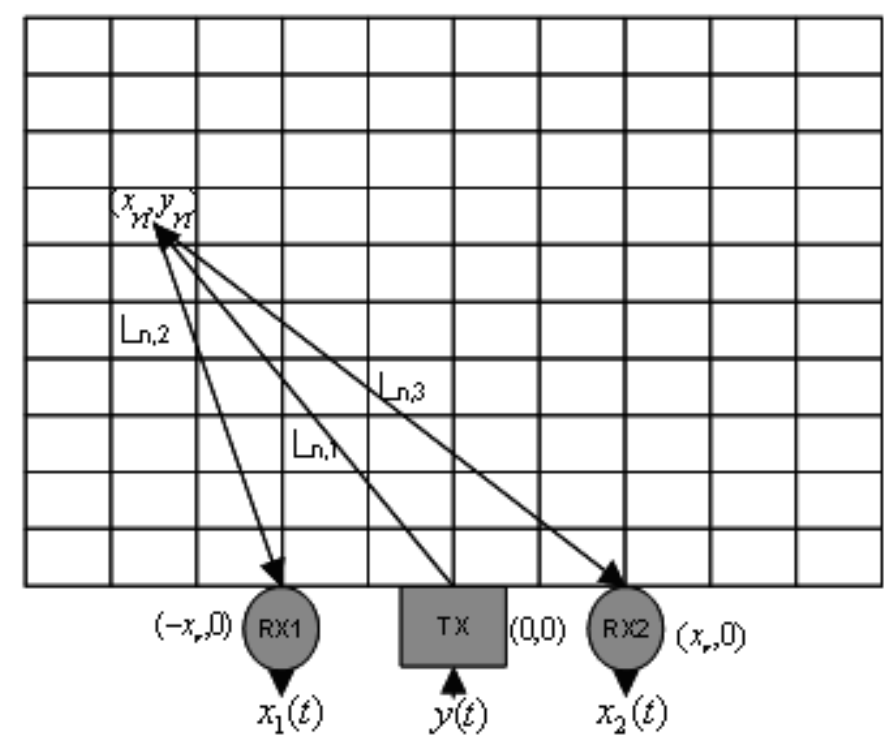

Figure 4: The position of the TX/RX antennas and the target in the $\mathrm{x}-\mathrm{y}$ planes. 
a)

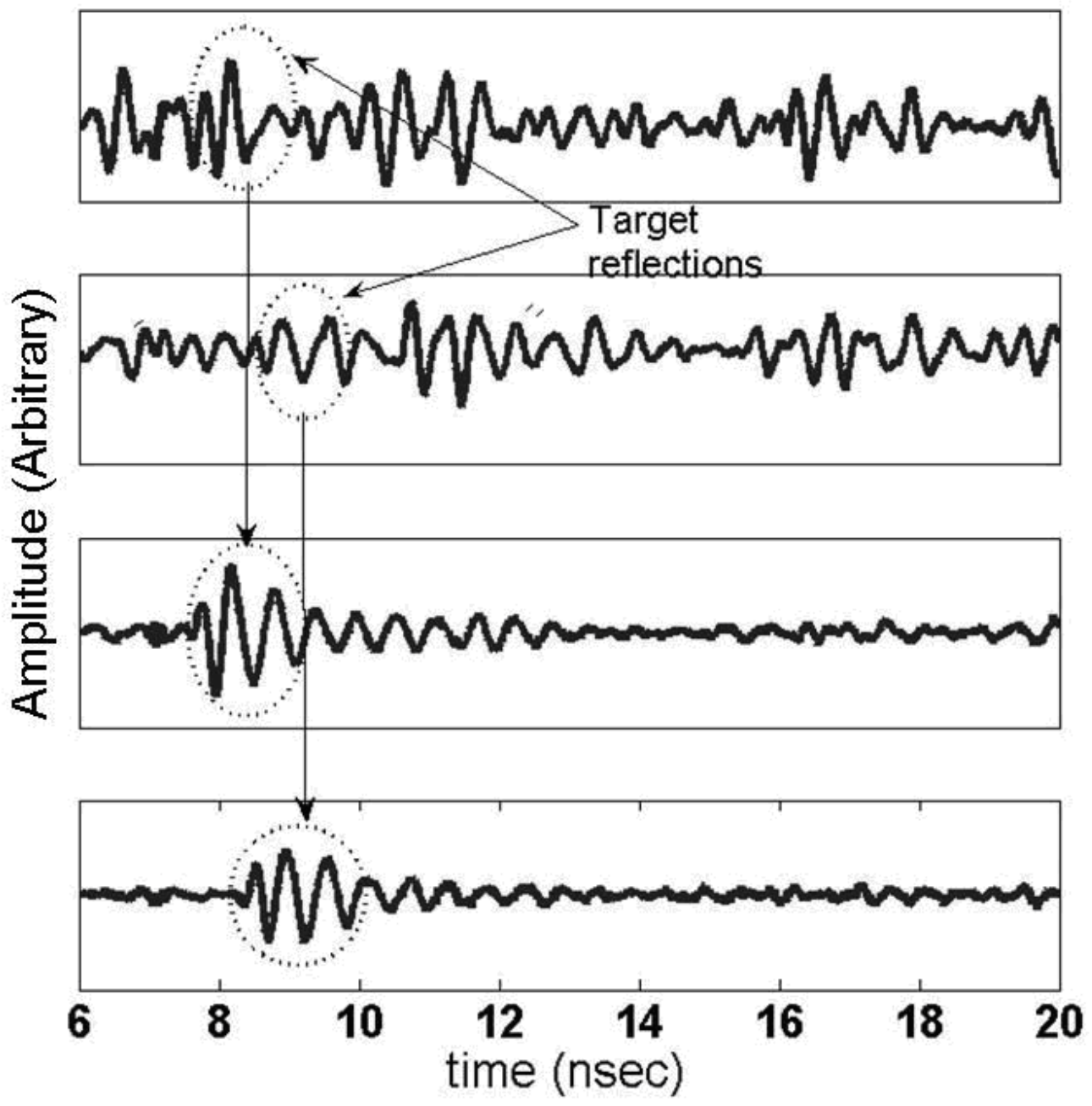

Figure 5: The received and calibrated waveforms. a) by receiver-1 b) by receiver-2 c) by receiver-1 after calibration d) by receiver-2 after calibration. 


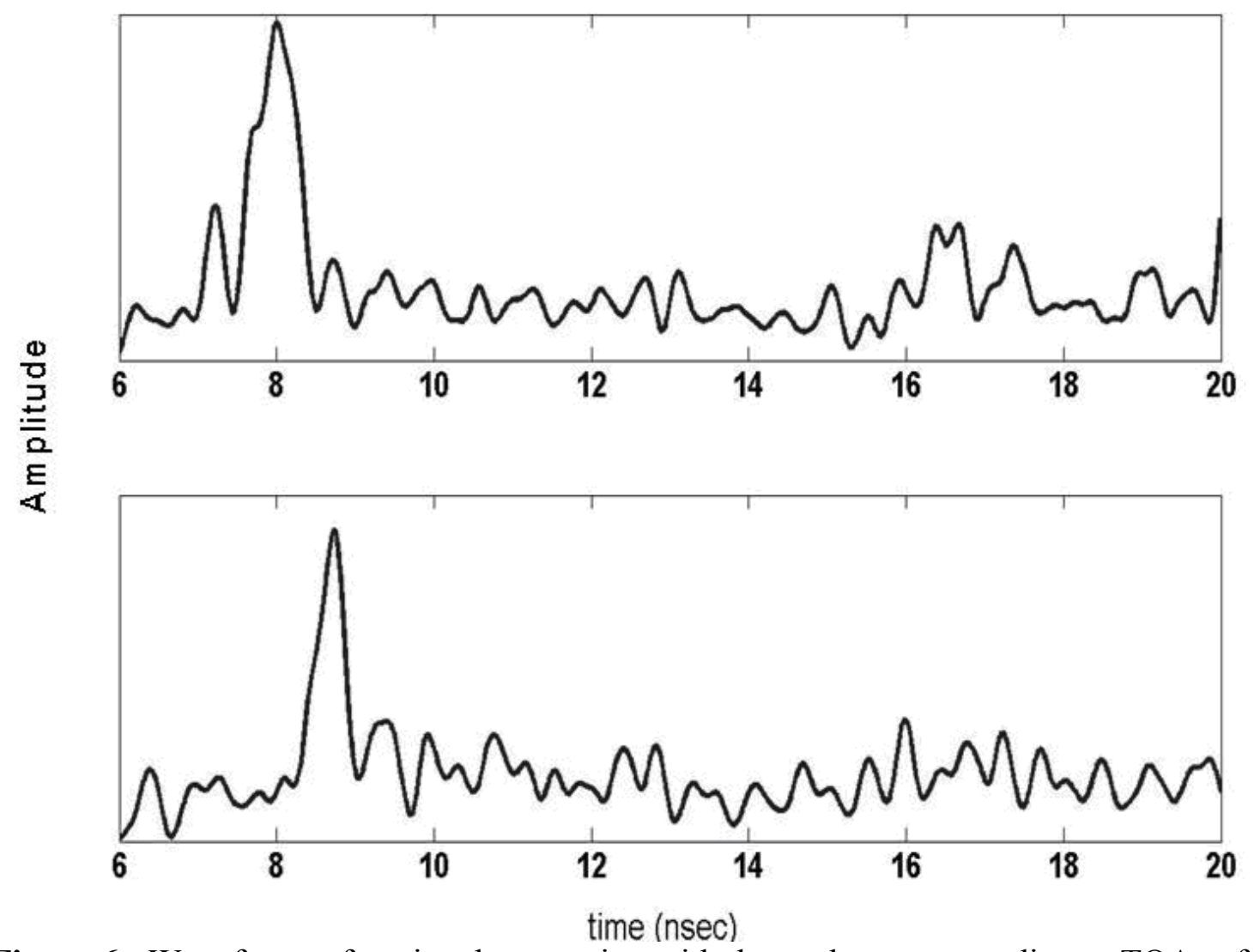

Figure 6: Waveforms after signal processing with the peaks corresponding to MOAs of the target. 


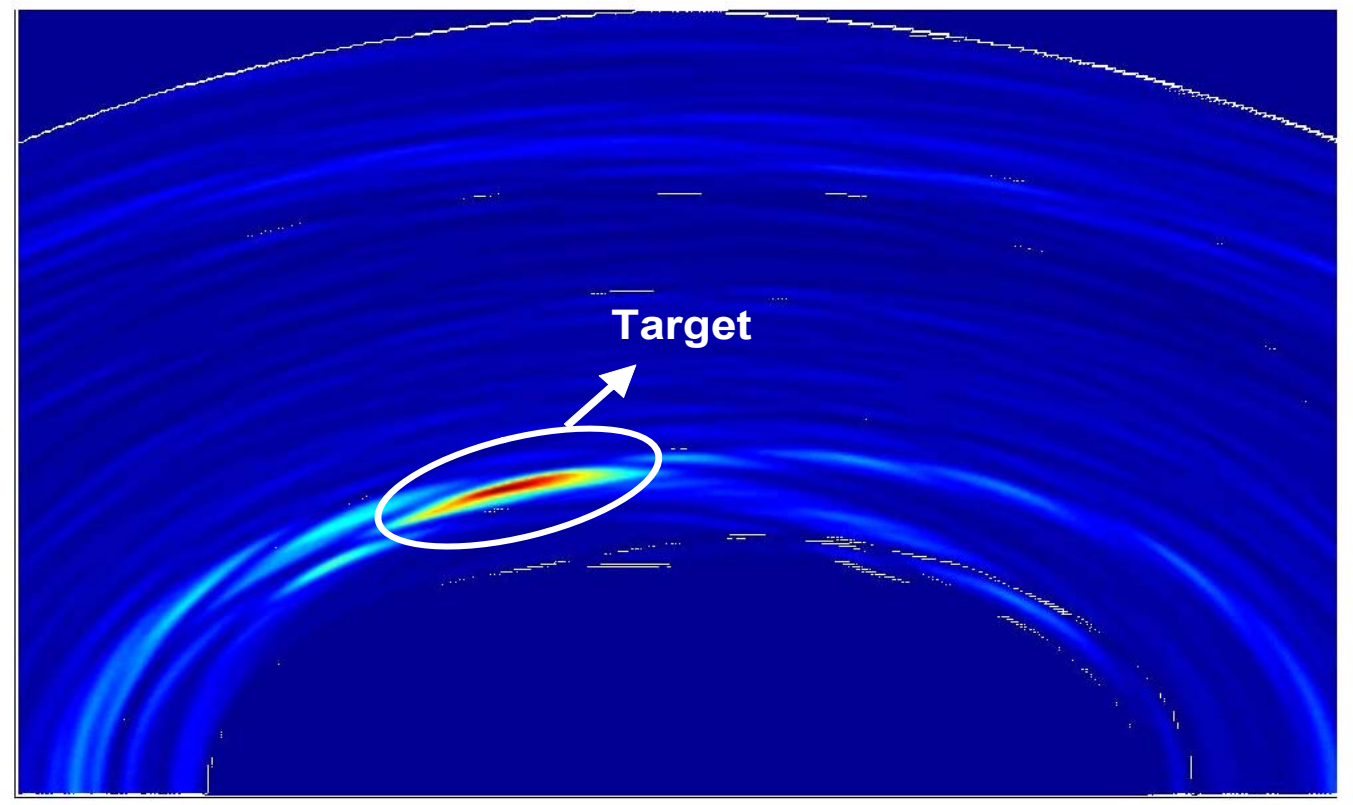

Figure 7: Weights are mapped to an intensity plot on an $x-y$ plane $(300 \mathrm{~cm} \times 300 \mathrm{~cm}$ with $1 \mathrm{~cm}$ grid). The bright region shows the position of the target. 


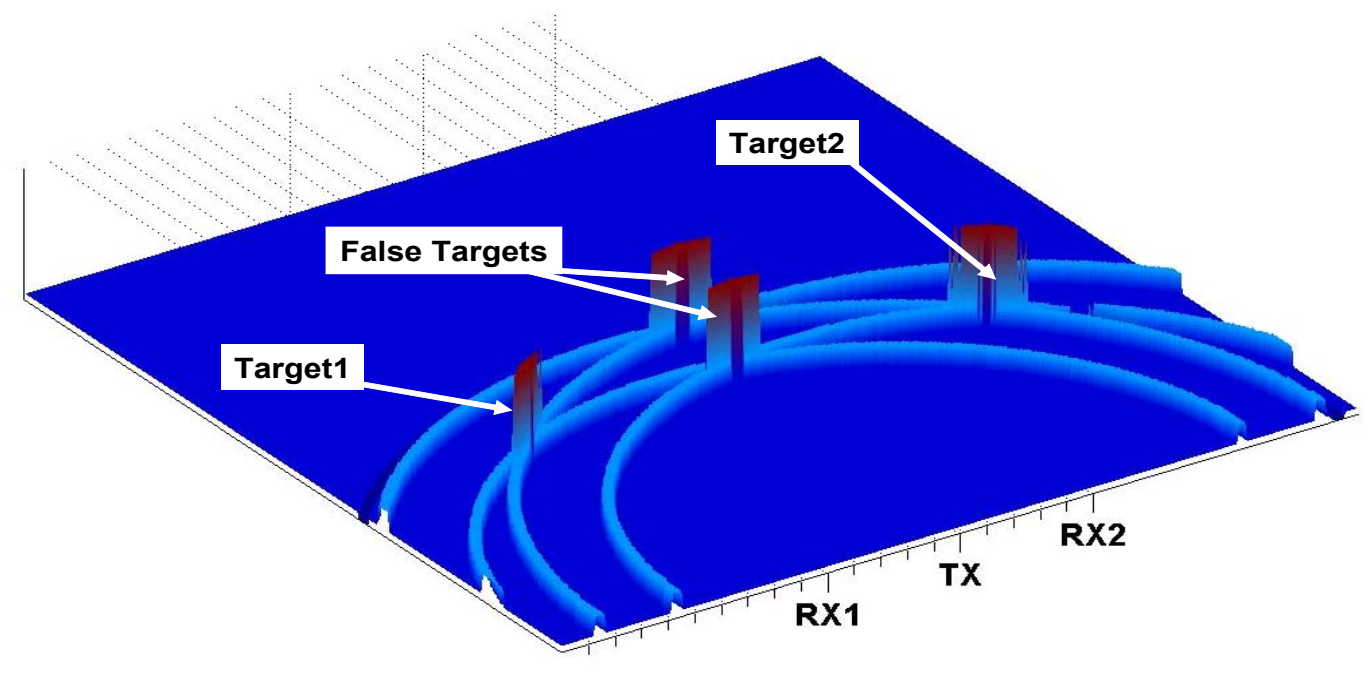

Figure 8: The real and false targets obtained by the intersection of four ellipses. 


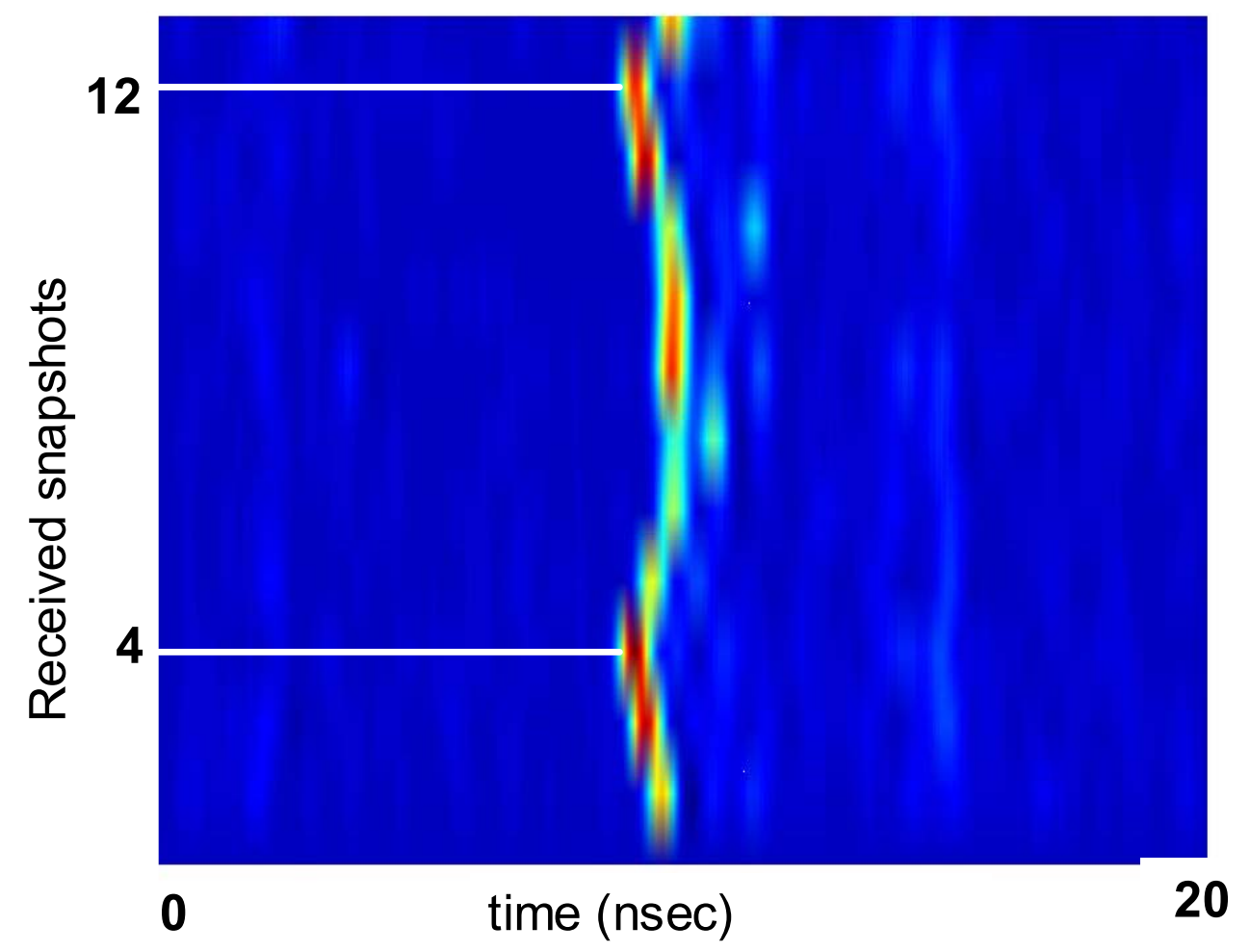

Figure 9: Intensity plot of the swinging ball with the period of the oscillatory motion as $3.04 \mathrm{sec}$. 


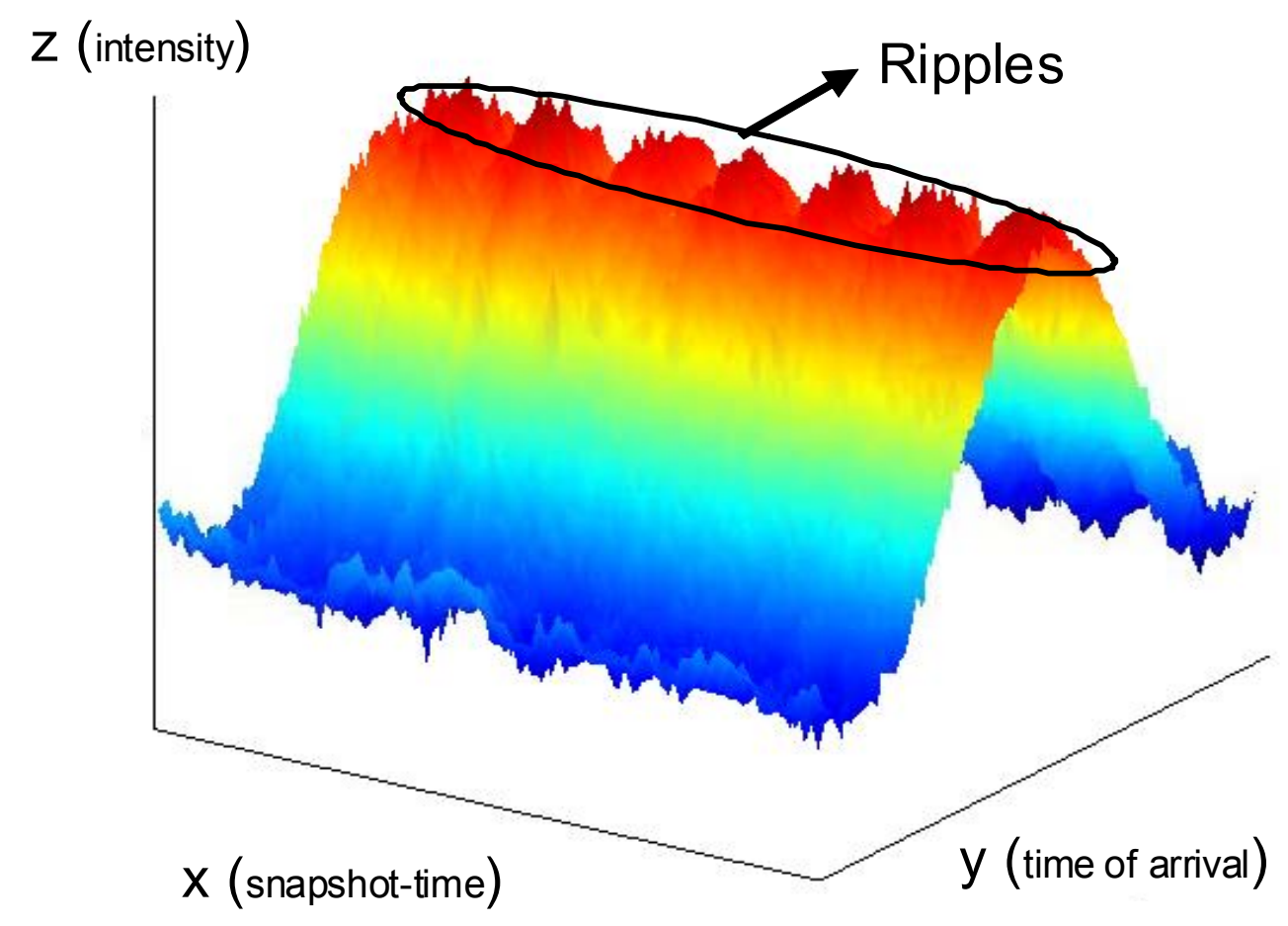

Figure 10: A 3-D intensity plot when a human is breathing behind a $23 \mathrm{~cm}$ thick brick wall with the ripples representing the respiratory activity. 


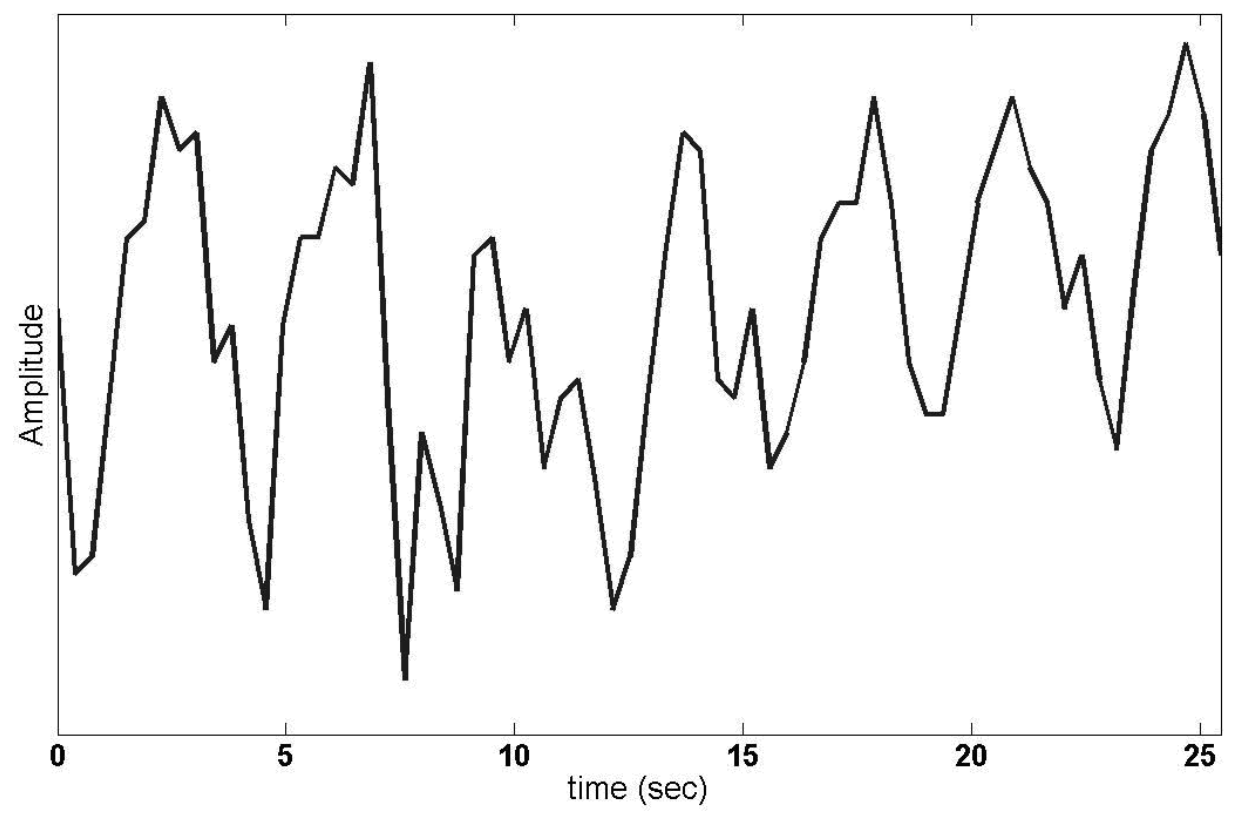

Figure 11: The respiration profile with the estimated $3.5 \mathrm{sec}$ period. 


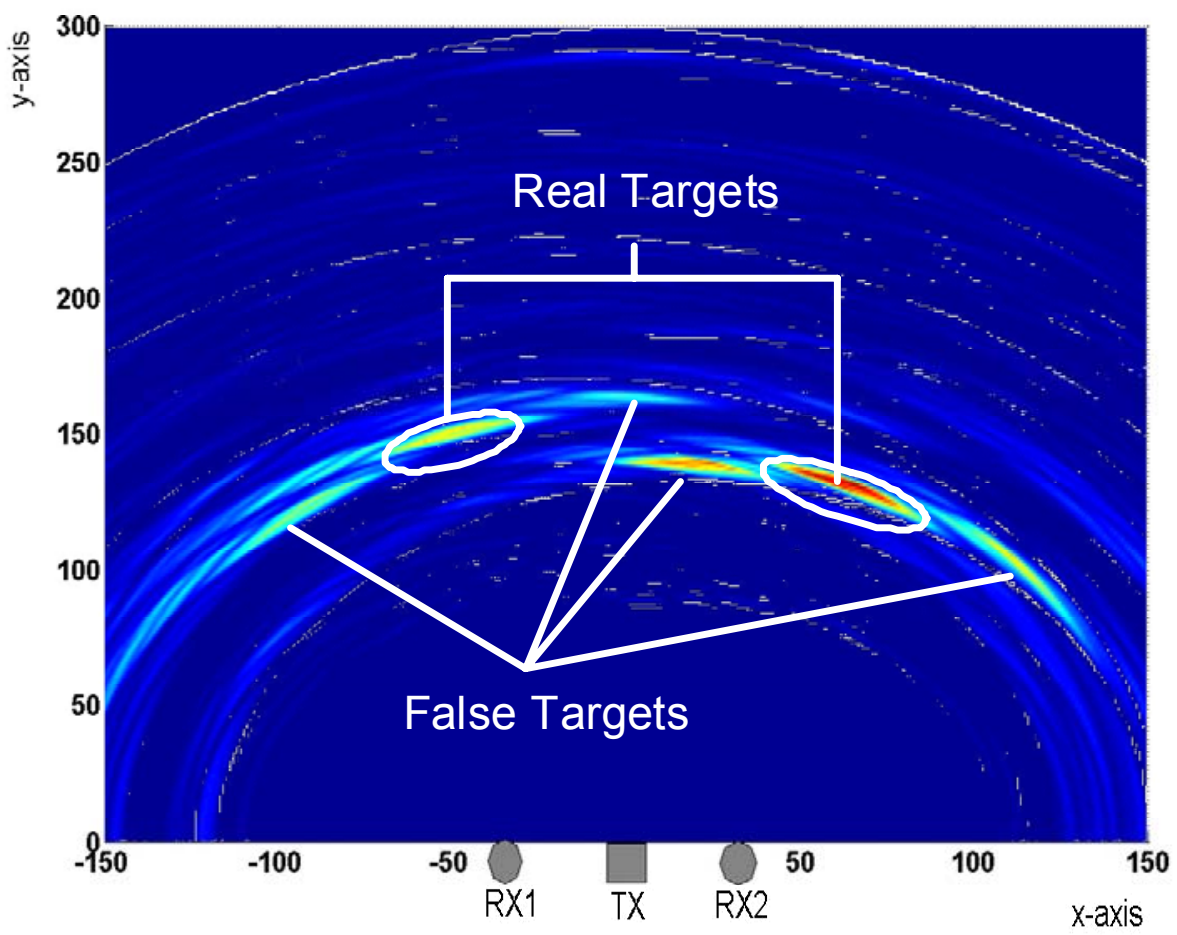

Figure 12: The intensity plot under multiple targets where a bottle and a metallic ball are placed in front of the radar. 


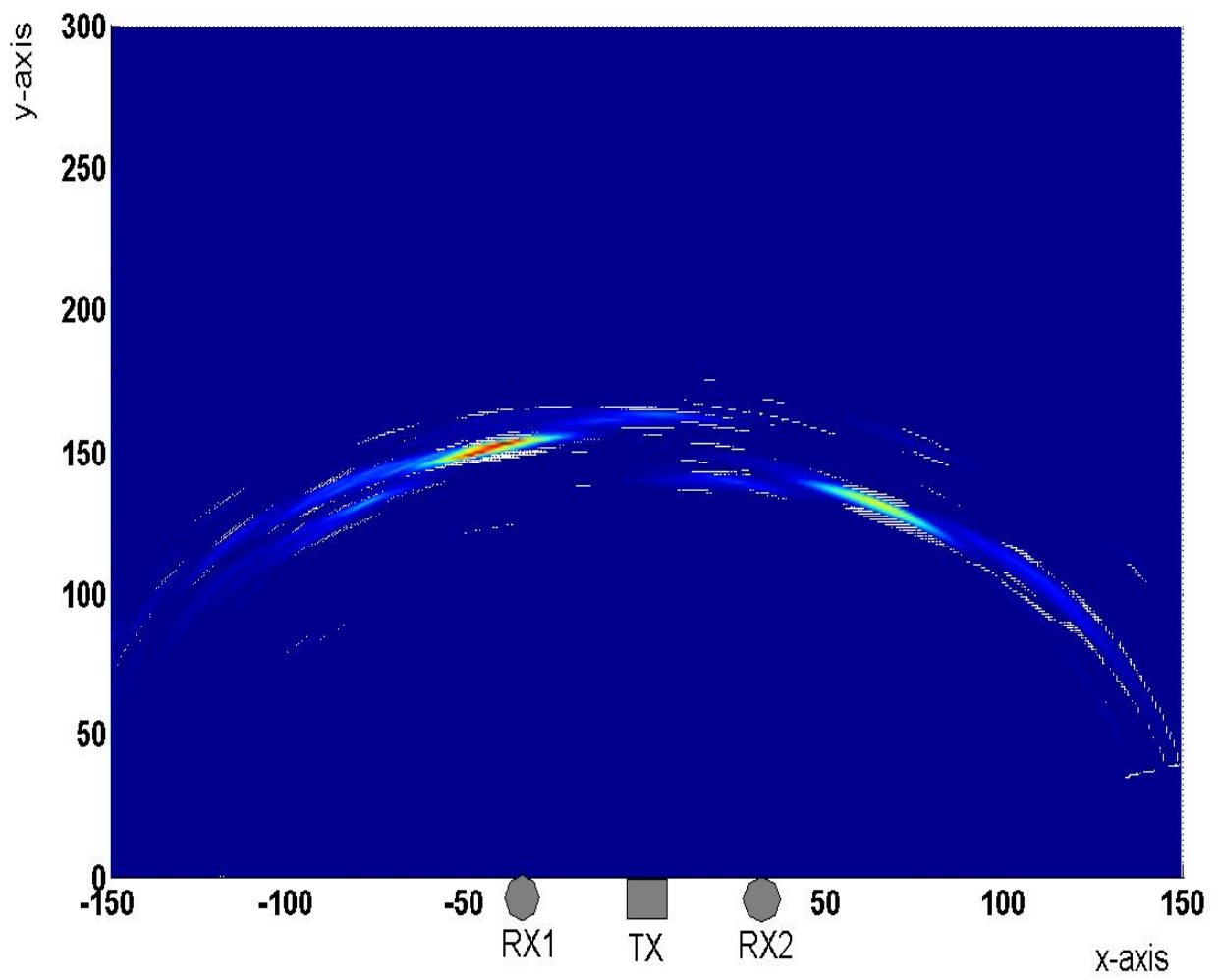

Figure 13: Elimination of the false targets by combining the results of two different positions of the antennas. 
Table 1: Radar operating parameters.

\begin{tabular}{lc}
\hline \hline Frequency Range & $1.85-6.3 \mathrm{GHz}$ \\
\hline Maximum Radiated Power & $110 \mathrm{~mW}$ \\
Minimum Pulse width & $100 \mathrm{psec}$ \\
Minimum Pulse Repetition Frequency & $31.3 \mathrm{MHz}$ \\
(PRF) & \\
Unambiguous Range & 4.5 meters \\
\hline \hline
\end{tabular}

